\title{
Optimal Decentralized Control for MIMO Systems
}

\author{
Ashraf M. Abdulhamed \\ B.Sc., and M.S.c., Electrical Engineering Dept. \\ Faculty of Engineering Minia University \\ El-Minia Egypt \\ Email: ashrafmed@hotmail.com
}

\author{
Ahmed A. M. El-Gaafary \\ Electrical Engineering Dept., \\ Faculty of Engineering Minia University \\ El-Minia Egypt \\ elgaafary@mu.edu.eg
}

\begin{abstract}
In this paper, an input decentralized control strategy is used to decompose MIMO system into many SISO sub systems. Placing the desired pole locations at a predetermined values by ITAE for each subsystem may leads to a slow overall system eigenvalues because of the interactions among each interconnected subsystem and others. Linear quadratic optimal technique is applied over subsystem level in order to shift the slowest obtained pole locations into much more stable places, which in turn shift the slowest overall system eigenvalues and guarantee a higher stability degree. This procedure is repeated many times until all the desired poles are achieved.
\end{abstract}

Keywords- Decentralized input control; Linear quadratic optimal technique (LQR); MIMO system; Pole placement.

\section{INTRODUCTION}

MIMO systems are considered as a complex control problem because of the interactions between all system inputs and outputs. This study introduces in detail a decentralized input control technique, in which the MIMO plant is decomposed into a set of SISO plants, and so the complex control problem can be divided into individual sub problems that have some interactions among each other, at this time it becomes easier to apply the classical control techniques over subsystem level [1,2].

In a decentralized control, each subsystem contains a local controller, which can be of different type, and has access to local sensors only $[1,3]$. These all-independent controllers together represent a decentralized controller, and the failure of one local controller does not necessarily lead to overall system instability.

The desired closed loop poles play a major role in subsystem as well as overall system stability. This study aims to investigate the possibility of using the optimally determined poles by ITAE. These poles are obtained by minimizing the integral of the time multiplied by the absolute value of the error, and how much the resulted poles of the overall system deviated from its nominal placed values for each decoupled subsystem, which affect subsystem as well as overall system stability.
Pole placement technique is used in control over a wide range and desired pole selection is a very important topic, $\mathrm{Yu}$ Zhang et al [4], designed PI controller to achieve dominant poles for multi loop system by a root trajectory method. In [5] Lavaei and Aghdam applied pole placement with decentralized proper interconnected systems, a set of decentralized controllers were designed to achieve desired pole locations for the modified decomposed subsystems. The desired closed loop poles were initially chosen by Alavian and Rotkowitz [6] for decentralized control by the use of the poles from the optimal centralized controller, then optimization methods were used to select poles from the candidates, finally they used Taylor approximation to adjust these poles to improve system performance. Erol, and Iftar [7], used an approach based on continuous pole placement algorithm; they proposed a decentralized pole assignment algorithm.

Linear quadratic optimal systems based on predetermined eigenvalues is an important manner. Alternative methods are developed by [8-11] to construct the weighting matrix $Q$ that enable the user to shift undesired system eigenvalues (unstable or slow) ones into a pre-specified (stable or faster) ones. The process can be repeated more and more until all the desired pole locations are placed.

\section{DECENTRALIZED INPUT CONTROL PROBLEM}

Consider a system $\mathbf{p}$ represented by the linear differential equation (1)

$$
\dot{z}=\hat{A} z+\hat{B} u
$$

where, $z \in R^{n}, u \in R^{s}, A \in R^{n \times n}, B \in R^{n \times s}$, this system can be decomposed into $\boldsymbol{r}$ dynamic elements.

$$
\dot{z}_{p}=\hat{A}_{p} z_{p}+\sum_{\substack{q=1 \\ q \neq p}}^{r} \hat{A}_{p q} z_{q}+\hat{B}_{p} u, \quad p=1,2, \ldots, r
$$

where, $z_{p} \in R^{l} p$, and $z=\left(z_{1}{ }^{T}, z_{2}{ }^{T}, \ldots, z_{r}{ }^{T}\right)^{T}, \quad n=\sum_{p=1}^{n} l_{p}$, such that all pairs $\left(\hat{A}_{p}, \hat{B}_{p}\right)$ are controllable, that is the $l_{p} \times l_{p s}$ matrix (3) has rank equal to $l_{p}[12,13]$. 


$$
\left[\begin{array}{lllll}
\hat{B}_{p} & \hat{A}_{p} \hat{B}_{p} & \hat{A}_{p}{ }^{2} \hat{B}_{p} & \cdots & \hat{A}_{p}^{p-1} \widehat{B}_{p}
\end{array}\right]
$$

A controllable system can be forced to satisfy any desired set of poles with the appropriate linear feedback [14]. For MIMO system, a subsystem or equivalently the pair $(A, B)$ is completely state controllable if there is an unconstrained control $u(t)$ that can transfer any initial state $x\left(t_{0}\right)$ to any other desired location $x(t)$ in a finite time, $t$ [12]. In this strategy the controllability of the individual subsystems is only required as a condition for control $[2,15]$.

A linear transformation is used to get $\bar{B}_{p}$ as the form of (4), in which $\bar{b}_{i}^{p} \in R^{n_{p i}}, l_{p}=\sum_{i=1}^{s} n_{p i}$

$$
\bar{B}_{p}=\left[\begin{array}{ccc}
\bar{b}_{1}^{p} & \cdots & 0 \\
\vdots & \ddots & \vdots \\
0 & \cdots & \bar{b}_{s}^{p}
\end{array}\right], \quad \bar{b}_{i}^{p}=\left[\begin{array}{c}
1 \\
0 \\
\vdots \\
0
\end{array}\right]
$$

The system represented by (2) is transformed into the form of (5), with the linear nonsingular transformation of (6)

$$
\begin{gathered}
\dot{\bar{z}}_{p}=\bar{A}_{p} \bar{z}_{p}+\sum_{\substack{q=1 \\
q \neq p}}^{r} \bar{A}_{p q} \bar{z}_{q}+\bar{B}_{p} u, p=1,2, \ldots, r \\
\bar{z}_{p}=Q_{p}{ }^{-1} z_{p}
\end{gathered}
$$

Where

$$
Q_{p}=\left[\hat{b}_{1}^{p}, \ldots, \quad \hat{A}_{p}^{n_{p s^{-1}}} \hat{b}_{1}^{p} ; \quad \ldots ; \quad \hat{b}_{s}^{p}, \ldots, \quad \hat{A}_{p}^{n_{p s^{-1}}} \hat{b}_{s}^{p}\right]
$$

And $\hat{b}_{i}^{p} \in R^{l_{p}}, i=1,2, \ldots, s$ are the columns of the matrix $\hat{B}_{p}$, $\left(n_{p 1}, n_{p 2}, \ldots, n_{p s}\right)$ are the dimensions of the sub-sub systems.

Regrouping Sub Systems: Now we can regroup the $\bar{z}_{p i}{ }^{\prime} s$ of each of $\boldsymbol{p}$ element which correspond to the same input $u_{i}$, and form the $i^{\text {th }}$ subsystem with the state $x_{i}(t) \in R^{n_{i}}$, such that $x_{i}=\left(\bar{z}_{1 i}{ }^{T}, \bar{z}_{2 i}{ }^{T}, \ldots, \bar{z}_{r i}{ }^{T}\right)^{T}$ and $n_{i}=\sum_{p=1}^{r} n_{p i}$.

This process of grouping yields finally the representation of the system $\mathrm{P}$ as composed of interconnected sub systems $S_{i}$ described by (8).

$$
\dot{x}_{i}=A_{i} x_{i}+\sum_{\substack{j=1 \\ j \neq i}}^{s} A_{i j} x_{j}+b_{i} u_{i}, i=1,2, \ldots, s
$$

To compute the matrices $A_{i}, A_{i j}$, and the vector $b_{i}$ from $\bar{A}_{p}, \bar{A}_{p q}, \bar{B}_{p}$, let us denote $\bar{z}=\left(\bar{z}_{1}{ }^{T}, \bar{z}_{2}{ }^{T}, \ldots, \bar{z}_{r}{ }^{T}\right)^{T}$ and $x=$ $\left(x_{1}{ }^{T}, x_{2}{ }^{T}, \ldots, x_{s}{ }^{T}\right)^{T}$, the state vectors of the overall systems corresponding to (5) and (8), respectively, then the grouping process is carried out by the nonsingular linear transformation

$$
x=P \bar{z}
$$

where the permutation matrix $P$ has the block form $P=$ $\left(P_{1}^{T}, P_{2}{ }^{T}, \ldots, P_{S}^{T}\right)^{T}$, and the $i^{\text {th }}$ block $P_{i} \in R^{n_{i} \times n}$ is defined by (10)

$$
P_{i}=\left[\begin{array}{cccc|ccccccc}
0 & \cdots & 0 & I_{1} & \cdots & \cdots & 0 & 0 & 0 & . . & 0 \\
0 & \cdots & 0 & d & \cdots & \cdots & I_{2 i} & 0 & 0 & \cdots & 0 \\
0 & \cdots & 0 & 0 & \cdots & \cdots & \cdots & \cdots & 0 & . . & 0 \\
0 & \cdots & 0 & 0 & \cdots & \cdots & \cdots & I_{r i} & 0 & \cdots & 0
\end{array}\right]
$$

where $I_{p i}$ is the $n_{p i} \times n_{p i}$ identity matrix and the zero matrices in (10) have the appropriate dimensions. Now we can write (5) as

$$
\dot{\bar{z}}=\bar{A} \bar{z}+\bar{B} u
$$

From (9)-(11), the transformed system is

$$
\dot{x}=A x+B u
$$

With the transformation

$$
A=P \bar{A} P^{T}, \quad B=P \bar{B}
$$

And

$$
B=\left[\begin{array}{ccc}
b_{1} & \cdots & 0 \\
\vdots & \ddots & \vdots \\
0 & \cdots & b_{s}
\end{array}\right], \quad b_{i}=\left[\begin{array}{c}
b_{i}^{1} \\
b_{i}^{2} \\
\vdots \\
b_{i}^{r}
\end{array}\right]
$$

Finally, the system resulted in (12) is defined as $\boldsymbol{S}$ interconnected input-decentralized subsystems, for the system described by (8).

The first system transformation is performed over the subsystem level, and the last transformation is performed over the overall system level, and it aims to regroup the components of the state vector.

\section{STABILIZING OF A DECENTRALIZED SYSTEM}

Input decentralized system can be stabilized by a multilevel control. Each decoupled subsystem can be stabilized separately using local controllers, which can provide a desired degree of stability, while global controllers are applied to enhance the overall system stability through reducing the effect of the interconnections among subsystems [15-17].

To stabilize the system given by (8), the decentralized multilevel control in (15) is applied

$$
u_{i}=u_{i}^{l}(t)+u_{i}^{g}(t)
$$

where, $u_{i}{ }^{l}(t)$ is the local controller and chosen as

$$
u_{i}^{l}(t)=-k_{i}^{T}(t) x_{i}
$$

With a constant vector $k_{i} \in R^{n_{i}}$, and $u_{i}{ }^{g}(t)$ is the global controller and chosen as 


$$
u_{i}^{g}(t)=-\sum_{j=1}^{s} k_{i j}{ }^{T} x_{j}
$$

where, $k_{i j} \in R^{n_{j}}$ are constant vectors.

Substituting (16), and (17) into (8) to get the overall system as (18)

$$
\begin{aligned}
\dot{x}_{i}{ }^{c}=\left(A_{i}{ }^{c}-b_{i}{ }^{c}\right. & \left.\left(k_{i}{ }^{c}\right)^{T}\right) x_{i}{ }^{c} \\
& +\sum_{\substack{j=1 \\
j \neq i \\
j \neq i}}\left(A_{i j}{ }^{c}-b_{i}{ }^{c}\left(k_{i j}{ }^{c}\right)^{T}\right) x_{j}{ }^{c}, i \\
& =1,2, \ldots, s
\end{aligned}
$$

Since each pair $\left(A_{i}, b_{i}\right)$ is controllable, and $k_{i}$ is calculated in order to place the eigenvalues of the decoupled subsystems $\left(A_{i}-b_{i} k_{i}\right)$ to its desired distinct stable locations, $-\sigma_{1}{ }^{i} \pm$ $j \omega_{1}{ }^{i}, \ldots,-\sigma_{p}{ }^{i} \pm j \omega_{p}{ }^{i}, \ldots,-\sigma_{p+1}^{i}, \ldots,-\sigma_{n_{i}-p}^{i}$ and $\sigma_{q}{ }^{i}>$ $0, q=1,2, \ldots, n_{i}-p$, and $0 \leq p \leq n_{i} / 2$

where, $\sigma_{q}$ is the real part of the desired pole, $\omega_{p}$ is the imaginary part and $n_{i}$ is the subsystem dimension.

Each decoupled subsystem in (19) is assumed of low order and control techniques can be applied to shift the undesired eigenvalues of these subsystems.

$$
\dot{x}_{i}{ }^{c}=\left(A_{i}^{c}-b_{i}{ }^{c}\left(k_{i}^{c}\right)^{T}\right) x_{i}^{c}, i=1,2, \ldots, s
$$

\section{A. Strong Coupled Case}

To stabilize a decentralized MIMO system the interconnected subsystems $\left(A_{i j}\right)$ in (8) is checked. If the upper diagonal of $\left(A_{i j}\right)$ is zero, then it is called a weakly coupled system, otherwise it is called a strongly coupled system [15].

To start stabilization process, (8) must be represented in canonical form (20) by using the transformation of (21).

$$
\left.\begin{array}{c}
\dot{x}_{i}{ }^{c}=A_{i}{ }^{c} x_{i}{ }^{c}+\sum_{\substack{j=1 \\
j \neq i}}^{s} A_{i j}{ }^{c} x_{j}{ }^{c}+b_{i}{ }^{c} u_{i}, i=1,2, \ldots, s \\
A_{i}{ }^{c}=\left(Q_{i}{ }^{c}\right)^{-1} A_{i} Q_{i}{ }^{c} \\
A_{i j}{ }^{c}=\left(Q_{i}{ }^{c}\right)^{-1} A_{i j} Q_{j}{ }^{c} \\
b_{i}{ }^{c}=\left(Q_{i}{ }^{c}\right)^{-1} b_{i}
\end{array}\right\}
$$

The canonical transformation matrix $Q_{i}$ is given by

$$
Q_{i}{ }^{c}=H_{i} \Lambda_{i}
$$

where

$$
\begin{gathered}
H_{i}=\left[\begin{array}{llll}
b_{i} & A_{i} b_{i} & \cdots & A_{i}^{n_{i}-1} b_{i}
\end{array}\right] \\
\text { B. Lyapunov Stability for The Interconnected Systems }
\end{gathered}
$$

To provide a Lyapunov function with the exact estimate of $\pi_{i}$ for each decoupled subsystem, a linear nonsingular transformation matrix $T_{i}$ is applied to $(19)[16,17,20]$ to diagonalize it as following

$$
\begin{gathered}
\tilde{x}_{i}{ }^{d}=T_{i}{ }^{-1} x_{i}{ }^{c} \\
T_{l} \tilde{x}_{l}{ }^{d}=\left(A_{i}{ }^{c}-b_{i}{ }^{c}\left(k_{i}{ }^{c}\right)^{T}\right) T_{i} \tilde{x}_{i}{ }^{d}
\end{gathered}
$$

where

And

$$
\dot{\tilde{x}}_{l}^{d}=\Lambda_{i} \tilde{x}_{i}^{d}
$$

$$
\Lambda_{i}=T_{i}^{-1}\left(A_{i}^{c}-b_{i}{ }^{c}\left(k_{i}{ }^{c}\right)^{T}\right) T_{i}
$$

Has the quasi diagonal form.

Diagonalize (18) with this transformation of (29) to get the system (28)

$$
\begin{gathered}
\dot{\tilde{x}}_{l}^{d}=\Lambda_{i}{\widetilde{x_{l}}}^{d}+\sum_{\substack{j=1 \\
j \neq i}}^{s}\left(A_{i j}{ }^{d}-b_{i}{ }^{d}\left(k_{i j}{ }^{d}\right)^{T}\right){\widetilde{x_{J}}}^{d}, i \\
=1,2, \ldots, s
\end{gathered}
$$

where

$$
\left.\begin{array}{c}
A_{i j}{ }^{d}=T_{i}{ }^{-1} A_{i j}{ }^{c} T_{j} \\
b_{i}{ }^{d}=T_{i}{ }^{-1} b_{i}{ }^{c} \\
\left(k_{i j}{ }^{d}\right)^{T}=\left(k_{i j}{ }^{c}\right)^{T} T_{j}
\end{array}\right\}
$$

To stabilize the MIMO system, each interconnected subsystem must be stabilized first [15-17,21]. The decomposition aggregation method can be applied to determine system stability, the stability condition is represented by (30)

$$
W=(-1)^{k}\left|\begin{array}{cccc}
-\pi_{1} & \xi_{12} & \cdots & \xi_{1 k} \\
\xi_{21} & -\pi_{2} & \cdots & \xi_{2 k} \\
\cdots & \cdots & \cdots & \cdots \\
\xi_{k 1} & \xi_{k 2} & \cdots & -\pi_{k}
\end{array}\right|>0
$$

where, $\xi_{i j}$ equal to:

$$
\begin{gathered}
\xi_{i j}=\sqrt{\lambda_{M}}\left[( A _ { i j } { } ^ { d } - b _ { i } { } ^ { d } ( k _ { i j } { } ^ { d } ) ^ { T } ) ^ { T } * \left(A_{i j}{ }^{d}\right.\right. \\
\left.\left.-b_{i}{ }^{d}\left(k_{i j}{ }^{d}\right)^{T}\right)\right]
\end{gathered}
$$

$\lambda_{M}$, is the maximum eigen value of the indicated matrix, and $\pi_{i}$ is the minimum real part of the eigenvalues of the diagonalized subsystem described by (20), so it is required to diagonalize the controlled interconnected subsystems before studying the overall system stability.

\section{Global Controller Gain}


linear nonsingular transformation matrix $T_{i}$ is applied to (19) to diagonalize it as following

$$
\tilde{x}_{i}^{d}=T_{i}^{-1} x_{i}^{c}
$$

Diagonalize (18) with this transformation of (33) to get the system (34)

$$
\left.\begin{array}{c}
A_{i j}{ }^{d}=T_{i}{ }^{-1} A_{i j}{ }^{c} T_{j} \\
b_{i}{ }^{d}=T_{i}{ }^{-1} b_{i}{ }^{c} \\
\left(k_{i j}{ }^{d}\right)^{T}=\left(k_{i j}{ }^{c}\right)^{T} T_{j}
\end{array}\right\}
$$

To calculate the global controller gain $k_{i j}{ }^{d}$, which is required to reduce the effect of the interconnected subsystems, the interconnection between subsystems is assumed to be zero in (34)

$$
\begin{gathered}
A_{i j}{ }^{d}-b_{i}{ }^{d}\left(k_{i j}{ }^{d}\right)^{T}=0 \\
\text { i.e., } A_{i j}{ }^{d}=b_{i}{ }^{d}\left(k_{i j}{ }^{d}\right)^{T} \\
k_{i j}{ }^{d}=\left[\left[\left(b_{i}{ }^{d}\right)^{T}\left(b_{i}{ }^{d}\right)\right]^{-1}\left[\left(b_{i}{ }^{d}\right)^{T}\left(A_{i j}{ }^{d}\right)\right]\right]^{T}
\end{gathered}
$$

To deduce the stability of the overall system, substitute the values of the optimal choice of $k_{i j}{ }^{d}$ in (36), into (34), the overall system becomes

$\dot{\tilde{x}}_{l}^{d}$

$=\Lambda_{i}{\tilde{x_{l}}}^{d}$

$+\left[I_{i}-b_{i}{ }^{d}\left[\left(b_{i}{ }^{d}\right)^{T}\left(b_{i}{ }^{d}\right)\right]^{-1}\left(b_{i}{ }^{d}\right)^{T}\right] \sum_{\substack{j=1 \\ j \neq i}}^{s} A_{i j}{ }^{d} \tilde{x}_{j}{ }^{d}, i$

$=1,2, \ldots, s$

\section{The Overall Controller Gain}

Through the process of stabilizing the MIMO system, the feedback control vector can be calculated using $k_{i}$ and $k_{i j}$ which are computed for the subsystem level, to obtain the control function $u(t)$ substituting (16), and (17) into (15), yields

$$
u(t)=-\left(k_{i}^{c}\right)^{T} x_{i}^{c}-\sum_{\substack{j=1 \\ j \neq i}}^{s}\left(k_{i j}{ }^{d}\right)^{T} T_{j}{ }^{-1} x_{j}^{c}
$$

$$
u_{i}(t)=-\left[\left(k_{i}^{c}\right)^{T}+\sum_{\substack{j=1 \\ j \neq i}}^{s}\left(k_{i j}{ }^{d}\right)^{T} T_{j}^{-1}\right] x^{c}
$$

where

$$
x^{c}=\left(Q^{c}\right)^{-1} P Q^{-1} Z
$$

Let $k$ equal to

Then

$$
k=k^{c}\left(Q^{c}\right)^{-1} P Q^{-1}
$$

$$
k^{c}=\left[\left(k_{i}^{c}\right)^{T}+\sum_{\substack{j=1 \\ j \neq i}}^{s}\left(k_{i j}{ }^{d}\right)^{T} T_{j}{ }^{-1}\right]
$$

where, $k^{c}$ can be expressed in detail as

$$
k^{c}=\left[\begin{array}{cccc}
\left(k_{1}{ }^{c}\right)^{T} & \left(k_{12}{ }^{d}\right)^{T} T_{2}{ }^{-1} & \ldots & \left(k_{1 s}{ }^{d}\right)^{T} T_{s}{ }^{-1} \\
\left(k_{21}{ }^{d}\right)^{T} T_{1}{ }^{-1} & \left(k_{2}{ }^{c}\right)^{T} & \ldots & \left(k_{2 s}{ }^{d}\right)^{T} T_{s}{ }^{-1} \\
\vdots & \vdots & & \vdots \\
\left(k_{s 1}{ }^{d}\right)^{T} T_{1}{ }^{-1} & \left(k_{s 2}{ }^{d}\right)^{T} T_{2}{ }^{-1} & \left(k_{s}{ }^{c}\right)^{T}
\end{array}\right]
$$

\section{E. The Effect of Local Controller Gain}

For the strong-coupled system, the effect of the local controller on the overall MIMO system stability can be derived. To derive a Lyapunov function with the exact estimate of $\pi_{i}$ for each decoupled subsystem, the value of global controller elements $k_{i j}{ }^{d}=0$, is substituted into (31) to yield

$$
\xi_{i j}=\sqrt{\lambda_{M}}\left[\left(A_{i j}{ }^{d}\right)^{T} *\left(A_{i j}{ }^{d}\right)\right]
$$

Equation (30) can be applied to check the system internal stability.

\section{DESIGN TECHNIQUE}

LQR is an optimal design technique which guarantees robustness, while pole placement technique gives the desired performance but does not guarantee robustness. In this procedure, a design of linear quadratic system with prescribed poles has been followed and applied to shift slow decoupled subsystem eigenvalues to defense the effect of interconnections, that leads to a much more stable overall system poles. This procedure was developed by Solheim [8]. The optimal gain is determined to minimize the cost function $J$ (45)

$$
J=\frac{1}{2} \int_{0}^{\infty}\left(x^{T} Q x+u^{T} R u\right) d t
$$

where, $k$ is given by (46) 


$$
k=R^{-1} B^{T} P
$$

where, $P$ is the unique, symmetric, positive definite solution to steady state Algebraic Ricatti Equation (ARE) (47)

$$
P A+A^{T} P-P B R^{-1} B^{T} P+Q=0
$$

To construct the weighting matrix $Q$ to shift the complex conjugate poles to complex places, (48) is used with the constraint in (49) for its internal elements

$$
\tilde{q}_{11}=\frac{2\left(\sigma^{2}-\alpha^{2}\right)-2\left(\omega^{2}-\beta^{2}\right)}{h_{11}+h_{22}}
$$

where $\alpha \pm j \beta$, are the old complex eigenvalues and $\sigma \pm j \omega$ are the desired complex eigenvalues

$$
\begin{aligned}
\left(\sigma^{2}+\omega^{2}\right)^{2}=( & \left.\alpha^{2}+\beta^{2}\right)^{2} \\
& +\tilde{q}_{11}\left(h_{11}+h_{22}\right)\left(\alpha^{2}+\beta^{2}\right) \\
& +\tilde{q}_{11}{ }^{2}\left(h_{11} h_{22}-h_{12}{ }^{2}\right)
\end{aligned}
$$

If it is required to shift complex conjugate to real places, (50) is used with the constraint in (51)

$$
\begin{gathered}
\tilde{q}_{11}=\frac{\sigma_{1}{ }^{2}+\sigma_{2}{ }^{2}-2\left(\alpha^{2}-\beta^{2}\right)}{h_{11}+h_{22}} \\
\sigma_{1}{ }^{2} \sigma_{2}{ }^{2}=\left(\alpha^{2}+\beta^{2}\right)^{2}+\tilde{q}_{11}\left(h_{11}+h_{22}\right)\left(\alpha^{2}+\beta^{2}\right) \\
+\tilde{q}_{11}{ }^{2}\left(h_{11} h_{22}-h_{12}{ }^{2}\right)
\end{gathered}
$$

if it is required to shift the real eigenvalues to real places, (52) is used

$$
\tilde{q}_{j j}=\frac{S_{j}^{2}-\lambda_{j}^{2}}{h_{j j}}
$$

where $S_{j}$ is the desired real eigenvalue and $\lambda_{j}$ is the old real one.

\section{SYSTEM STUDY}

Consider the system represented by (54).

$$
\dot{z}=\left[\begin{array}{lllll}
1 & 3 & 4 & 5 & 6 \\
0 & 5 & 2 & 4 & 1 \\
2 & 4 & 0 & 0 & 6 \\
2 & 0 & 2 & 1 & 6 \\
2 & 2 & 3 & 0 & 1
\end{array}\right] z+\left[\begin{array}{ll}
1 & 2 \\
0 & 4 \\
2 & 0 \\
0 & 2 \\
1 & 3
\end{array}\right] u
$$

This system with $n=5, s=2, s$ (number of inputs), can be decomposed into two $(r=2)$ dynamic elements (subsystems)

$$
\left.\begin{array}{l}
\dot{z}_{1}=\left[\begin{array}{ll}
1 & 3 \\
0 & 5
\end{array}\right] z_{1}+\left[\begin{array}{lll}
4 & 5 & 6 \\
2 & 4 & 1
\end{array}\right] z_{2}+\left[\begin{array}{ll}
1 & 2 \\
0 & 4
\end{array}\right] u \\
\dot{z}_{2}=\left[\begin{array}{ll}
2 & 4 \\
2 & 0 \\
2 & 2
\end{array}\right] z_{2}+\left[\begin{array}{lll}
0 & 0 & 6 \\
2 & 1 & 6 \\
1 & 1 & 1
\end{array}\right] z_{1}+\left[\begin{array}{ll}
2 & 0 \\
0 & 2 \\
1 & 3
\end{array}\right] u
\end{array}\right\}
$$

So that the dimensions of subsystems 1 and 2 are respectively, $L_{1}=2, L_{2}=3$.

Controllability of both pairs $\left(\hat{A}_{1}, \hat{B}_{1}\right)$ for subsystem 1 and ( $\hat{A}_{2}, \hat{B}_{2}$ ) for subsystem 2 , are checked and the two subsystems are completely state controllable.

To transform the system to that form in (5) we can use the transformation represented by (6)-(8), to get the two converted sub systems, which can be collected again to present the overall system in (55)

$$
\begin{gathered}
\dot{\bar{z}}=\left[\begin{array}{ccccc}
1 & 4 & 11.5 & 86.5 & 22.5 \\
0 & 5 & 1.25 & 14.75 & 2.75 \\
0.455 & 8.91 & 0 & -4.1 & -0.82 \\
0.182 & 0.3636 & 21 & 8.363 & 3.27 \\
0.091 & 0.182 & 0 & -9.82 & -6.36
\end{array}\right] \bar{z} \\
+\left[\begin{array}{ll}
1 & 0 \\
0 & 1 \\
1 & 0 \\
0 & 0 \\
0 & 1
\end{array}\right] u
\end{gathered}
$$

Regroup the system in (55) using a determined permutation matrix (10). The matrices $\bar{B}_{1}$ and $\bar{B}_{2}$ have the form of (4), with the suggestion of

$$
\bar{b}_{1}^{1}=1, \bar{b}_{2}^{1}=1, \bar{b}_{1}^{2}=\left[\begin{array}{l}
1 \\
0
\end{array}\right], \text { and } \bar{b}_{2}^{2}=1
$$

and $n_{11}=1, n_{12}=1, n_{21}=2, n_{22}=1$. Therefore, $n_{1}=$ $n_{11}+n_{21}=3$ and $n_{2}=n_{12}+n_{22}=2$, the two subsystems are formed by regrouping the components of

$$
\bar{z}_{1}=\left(\bar{z}_{11}{ }^{T}, \bar{z}_{12}{ }^{T}\right)^{T}, \quad \bar{z}_{2}=\left(\bar{z}_{21}{ }^{T}, \bar{z}_{22}{ }^{T}\right)^{T}
$$

Calculated permutation matrices $P_{1}, P_{2}$ are used in order to get new subsystems with the states

$$
x_{1}=\left(\bar{z}_{11}{ }^{T}, \bar{z}_{21}{ }^{T}\right)^{T}, \quad x_{2}=\left(\bar{z}_{12}{ }^{T}, \bar{z}_{22}{ }^{T}\right)^{T}
$$

The resulted decentralized system is

$$
\begin{array}{r}
\dot{x}=\left[\begin{array}{ccccc}
1 & 11.5 & 86.5 & 4 & 22.5 \\
0.454 & 0 & -4.1 & 8.91 & -0.82 \\
0.182 & 1 & 8.36 & 0.36 & 3.27 \\
5 & 2.75 & 0 & 1.25 & 14.75 \\
0.182 & -6.36 & 0.09 & 0 & -9.82
\end{array}\right] x \\
+\left[\begin{array}{ll}
1 & 0 \\
1 & 0 \\
0 & 0 \\
0 & 1 \\
0 & 1
\end{array}\right] u
\end{array}
$$

This can be decomposed into two interconnected input decentralized subsystems. Right transformations can be 
checked by obtaining the same eigenvalues for the system in (54), (55), and (56).

To design a decentralized controller, the two interconnected subsystems are transferred in (56) into companion canonical form by following the preceding transformation steps in (21)(23).

The eigenvalues of the decoupled subsystem one are $\left[\begin{array}{lll}10.11 & 0.63 & -1.385\end{array}\right]$ and for subsystem two are [5.04-6.4] , and it is clear that these two subsystems are unstable.

\section{A. Pole Placement Design}

Now place the closed loop system poles according to the predetermined ITAE prototype values at natural frequency of $\omega_{n}=1 \mathrm{rad} / \mathrm{sec}[23,24]$. For the first subsystem they can be placed at $\left[\lambda_{1}=-0.7081, \lambda_{2,3}=-0.521 \pm j 1.068\right]$ and for the second subsystem are placed at $\left[\lambda_{4,5}=-0.707 \pm j 0.707\right]$, the feedback gains required to place system poles to these desired values can be determined to be

$k_{1}{ }^{c}=\left[\begin{array}{lll}-7.826 & 10.65 & 11.11\end{array}\right]$ and $k_{2}{ }^{c}=\left[\begin{array}{ll}33.3 & 0.0541\end{array}\right]$

The overall system eigenvalues are determined to be $E_{1 a}$ :

$$
E_{1 a}=\left[\begin{array}{llll}
-6.46 & 1.55 \pm j 6.36 & -1.6 & 1.8
\end{array}\right],
$$

It means that the overall system is unstable.

Global Controller Design: From (36), global controller can be designed to be:

$$
\begin{gathered}
k_{12}{ }^{d}=\left[\begin{array}{ll}
54.7 & 17.19
\end{array}\right] \text { and } \\
k_{21}{ }^{d}=\left[\begin{array}{lll}
-5.955 & 4.9 & -0.246
\end{array}\right]
\end{gathered}
$$

Computing the Overall Gain $k$ : To determine the overall gain $k,(41)$ and (42) are used to determine the overall gain $k$ and the overall system eigenvalues are computed and summarized in table. 1

Now the system becomes stable but one complex pair becomes very slow (near to the $j \omega$ axis), this pair may drive the system to de unstable under the effect of some disturbances or uncertainties. Also, the stability condition $W$ is checked for the controlled system with both local and global controllers and found $<0$, which means that the controlled system is internally unstable.

\section{B. LQR Design}

Now LQR procedures can be applied to shift the resulted undesired (slow poles) in Section IV into a more stable ones, in this design, a trial to enhance these locations by shifting the slow poles one by one or pair for each sub system individually.
The eigenvalues of subsystem I are $[-0.7081-0.521 \pm$ $j 1.608$ ] , so in order to move the two conjugate ones, $R=1$, and $Q_{11}$ is calculated as in Section IV is to be

$$
Q_{11}=\left[\begin{array}{ccc}
0 & 0 & 0 \\
0 & 13.2 & 0 \\
0 & 0 & 13.2
\end{array}\right]
$$

this value succeed to shift the system eigenvalues to $[-4-$ 1.36], which are accepted and more stable locations, another step can be applied to shift the third eigenvalue from -0.7081 to -6 , by

$$
Q_{12}=\left[\begin{array}{ccc}
658.6 & 0 & 0 \\
0 & 0 & 0 \\
0 & 0 & 0
\end{array}\right]
$$

The overall $Q$ for subsystem I is determined to be

$$
Q_{1 t}=\left[\begin{array}{ccc}
1069.7 & 1063.8 & 200.8 \\
1063.8 & 1074.6 & 214.6 \\
200.8 & 214.6 & 55.1
\end{array}\right]
$$

The resulted local controller gain required is $k_{1 q}=$ $\left[\begin{array}{lll}31.73 & 35.5 & 9.6\end{array}\right]$

For subsystem II, the eigenvalues are $[-0.7071 \pm$ $j 0.7071]$, so in order to move the complex pair, $R=1$, and $Q_{21}$, is calculated to be

$$
Q_{21}=\left[\begin{array}{cc}
13 & 0 \\
0 & 13
\end{array}\right]
$$

This value succeed to shift system eigenvalues to [ $-5-$ 1.04], another step can be applied to shift the resulted slowest eigenvalue from -1.04 to -8 , by

$$
Q_{22}=\left[\begin{array}{cc}
0 & 0 \\
0 & 906.6052
\end{array}\right]
$$

The overall Q for subsystem II is

$$
Q_{2 t}=\left[\begin{array}{cc}
1593.7 & 332.4 \\
332.4 & 88.9
\end{array}\right]
$$

With a local controller gain of $k_{2 q}=\left[\begin{array}{ll}38.934 & 11.58\end{array}\right]$

Global Controller Design: The global controller gain for both sub systems are computed to be:

$$
\begin{gathered}
k_{12 q}=\left[\begin{array}{ll}
54.7 & 17.19
\end{array}\right] \\
k_{21 q}=\left[\begin{array}{lll}
-5.955 & 4.9 & -0.246
\end{array}\right]
\end{gathered}
$$

The overall gain with LQR and the overall eigenvalues are computed and illustrated in table. 1. It is noted that the slowest controlled system pole is obtained to be at -1.45 which is faster. Stability condition $W$ is computed and illustrated in table.1. 


\section{CONCLUSIONS}

The use of the pre-determined poles by ITAE in a decentralized control using pole placement technique may lead to slow overall system eigenvalues, due to the effect of the interconnected subsystems up on the decoupled ones. These slow poles may drive the system into the instability region if it is affected by some disturbances or uncertainties, also these poles may fail to recognize system internal stability.
LQR technique can be applied over subsystem level for systems having slow poles in order to reduce the interconnections among subsystems through shifting the slowest subsystem eigenvalues into a more stable predetermined values, while keeping other poles unchanged. The shifted poles guarantee better overall system stability with no much more control effort than that required when using pole placement with nominal ITAE poles.

TABLE 1: DESIGN RESULTS

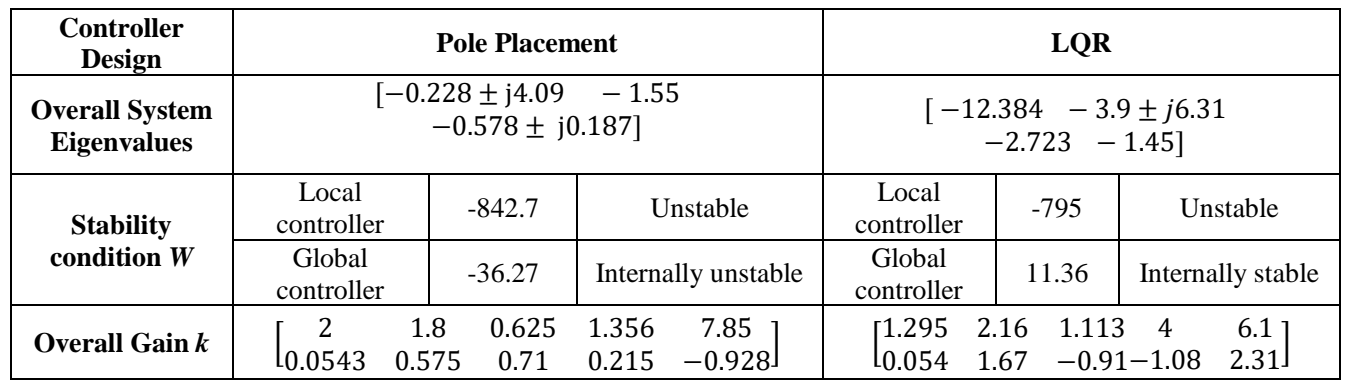

\section{REFERENCE}

[1] J. D. Polstonm, and L. Kentucky, " Decentralized adaptive control for uncertain linear systems: Technique with local fullstate feedback or local relative-degree-one output feedback", Master of Science, Mechanical Engineering, College of Engineering, Kentucky University, 2013.

[2] P. Chalupa, and V. Bobál, "Decentralized control of multivariable system using real time workshop", Department of Control Theory, Institute of Information Technologies, Tomas Bata University, Zlin.

[3] J. C. Sombria, J. S. Moreno, A. Visioli, and S. D. Bencomo, "Decentralised control of a quadruple tank plant with a decoupled event-based strategy ", IFAC Conference on Advances in PID Control, Brescia Italy, March 28-30, 2012.

[4] Y. Zhang, Q. G. Wang, and K. J. Astrom, "Dominant pole placement for multi-loop control systems", Elsevier Automatica, vol.38, no.7, pp. 1813-1820, 2002.

[5] J. Lavaei, and A. G. Aghdam, "Decentralized pole assignment for interconnected systems", American Control Conference, Washington, USA., June 11-13, 2008.

[6] A. Alavian, and M. Rotkowitz, "On the pole selection for Hooptimal decentralized control", American Control Conference, USA, pp. 5471-5476, July 2015.

[7] H. Erol, and A. Iftar, "Decentralized controller design by continuous pole placement for neutral time-delay system", SAGE Journals, Transactions of the Institute of Measurement and Control, vol.39, no.3, 2017.

[8] O. A. Solheim, "Design of optimal control systems with prescribed eigenvalues", Int. J. Control, vol.15, no.1, pp. 143$160,1972$.

[9] S. A. Ghoreishi, M. A. Nekoui and S. O. Basiri, "Optimal design of LQR weighting matrices based on intelligent optimization methods", International Journal of Intelligent Information Processing, vol.2, no., March 2011.

[10] M. Raluca, Stefanescu, C. L. Prioroc, and A. M. Stoica, "Weighting matrices determination using pole placement for tracking maneuvers ", U.P.B. Sci. Bull., Series D, vol.75, no.2, 2013
[11] V. Kučera, and J. Cigler, "Optimal control systems with prescribed eigenvalues", 4th International Symposium on Communications, Control and Signal Processing, ISCCSP, Limassol, Cyprus, 3-5 March 2010.

[12] S. Elizabeth, and R. Jothilakshmi, "Observability and controllability of MIMO control systems via difference equations", International Journal of Computer Applications, vol.62, no.1, pp37-42, 2013.

[13] R. L. Williams, and D. A. Lawrence, Linear State Space Control Systems. John Wiley \& Sons, Hoboken, New Jersey, 2007.

[14] V. Nath, and R. Mitra, "Robust pole placement using linear quadratic regulator weight selection algorithm", International Journal of Scientific Research Engineering \& Technology, vol. 3, no.3, pp 329-333, 2014.

[15] D. D. Siljak, Large Scale Dynamic Systems Stability and Structure. Elsevier North-Holland, Inc., 1978.

[16] V. Kučera," Decoupling optimal controllers", 18th International Conference on Process Control, Tatranská Lomnica, Slovakia, June 14-17, 2011, pp. 400-407.

[17] B. Labibi, B. Lohmann, A. K. Sedigh, and P. J. Maralani, "Sufficient condition for stability of decentralized control", Electronic Letters. vol.36, no.6, 16th March 2000

[18] G. F. Franklin, J. D. Pawell, and A. E. Naeini, Feedback Control of Dynamic Systems. fourth edition, Printic-Hall Inc., 2002.

[19] F. G. Martins, "Tuning PID controllers using the ITAE criterion", Int. J. Engng Ed., vol. 21, no.5, pp. 867-873, 2005.

[20] Y. Ma, Q. Zhang, and A. X. Zhang, "Decentralized output feedback robust control for a class of uncertain nonlinear circlelinked large-scale composite systems", Int. Journal of Information and Systems Sciences, vol.2, no.1, pp.20-30, 2006.

[21] D. D. Siljak, and D. M. Stipanovic, "Robust stabilization of nonlinear systems: The LMI approach", Mathematical Problems in Engineering, OPA Overseas Publishers Association N.V Singapore vol.6, pp.461-493, 2000 . 
Faculty of Energy Engineering - Aswan University - Aswan - Egypt 